growth factor receptor Flk-1 utilize related immunoglobulin domains for the binding of their respective ligands $\mathrm{s}^{6,7}$.

Their studies also compliment the identification of the critical amino acids of the neurotrophins that are required not only for neurotrophin-Trk binding, but also to convey specificity of Trk activation ${ }^{8}$. The crystal structure of NGF has been solved, and models of neurotrophin-Trk interactions have been proposed ${ }^{9}$. Thus, mutation of specific residues of NGF can render it ineffective in binding to all Trks, whereas directed mutagenesis of other amino acids of NT-3 can result in a "pan-neurotrophin" that can effectively bind and activate all three classes of Trk receptor ${ }^{8,10}$. The data from these previous reports, together with the current work of Holden and coworkers should produce a windfall of information for researchers in structure-based drug design and for predicting peptides capable of activating Trk receptors.

Besides the IgC2-like domains, the region between the transmembrane and the $\operatorname{IgG}$ domain nearest to the membrane may also be critical for binding of neurotrophins $s^{4,11}$. Another extracellular motif, the second leucine-rich repeat, has been reported to be sufficient for binding to NGF in the absence of the IgG domain ${ }^{12}$. Although there is a debate about the relative importance of the IgG domain versus the leucine-rich repeat, the differences may lie in the type of biochemical assay and the state of the recombinant proteins. However, the results suggest there is a potential for multiple interactions between receptor and neurotrophin, which is dependent upon the conformational status of each protein. Indeed, contacts between NGF and Trk A are present in the $\beta$-hairpin loops, and the amino and carboxyl termini of NGF. The intrinsic flexibility of these regions suggests that conformational changes may occur on formation of a productive NGF-Trk complex?.

Unlike many other ligand-receptor systems that utilize highly limited patterns of tissue expression to convey selectivity of action, the neurotrophins and their receptors are highly expressed in contiguous regions of the brain. These observations suggest that the selectivity of neurotrophin action may be regulated in the specific affinities of ligand -receptor interaction. Therefore, the accuracy in identifying the appropriate binding domains of Trk receptors that convey selectivity of neurotrophin binding will be critical for the eventual development of "docking" peptides that utilize a common Trk binding site, but display selectivity in activation of only a single class of Trk receptor.

Further studies of this kind will address the issue of what residues determine neurotrophin specificity. The current findings open the prospect of mimicking neurotrophins with small molecules that may display therapeutic potential in diseases ranging from Alzheimer's disease and amyotrophic lateral sclerosis to the ameloriation of pain pathways. Drug design and modeling are now within the realm of reality, as the regions responsible for neurotophin-receptor binding are now being defined to a small and accessible domain.

1. Holden, P.H., et al. 1997. Nature Biotechnology
15:668-672.

2. Lewin, G. and Barde, Y.-A. 1996. Annu. Rev. Neurosci. 19:289-317.

3. Perez, P. et al. 1995. Mol. Cell. Neurosci. 6:97-105.

4. Urfer, R. et al. 1995. EMBO J. 14:2795-2805.

5. Tannahill, L., Klein, R., and Schachner, M. 1995. Eur. J. Neurosci. 7:1424-1428.

6. Blechman, J.M. et al. 1995. Cell 80:103-113.

7. Davis-Smyth, T., Chen H., Park, J. et al. 1996. EMBO J. 15:4919-4927.

8. Ibanez, C.F. 1995.Trends Biotechnol. 13:217-227.

9. McDonald, N.Q. and Chao, M.V. 1995. J. Biol. Chem 270:19669-19672.

10. Urier, R. et al. 1994. EMBO J. 13:5896-5909.

11. MacDonald, J.I. and Meakin, S.O. 1996. Mol. Cell. Neurosci. 7;371-390.

12. Windisch, J.M., Steiner, M.R., and Schneider, R.J. 1995. Biol. Chem. 270:28133-28138.

\title{
Evolution goes for GOLD in silico
}

\section{Clare Sansom}

Several techniques call on evolution to discover or adapt potential drug molecules. Now, similar evolutionary principles are being applied in silico. GOLD-genetic optimization for ligand docking-(Jones et al., J. Mol. Biol. 267:727-748, 1997) is a program developed by Gareth Jones at the University of Sheffield (Sheffield, UK) in collaboration with Glaxo Wellcome (London) and the Cambridge Crystallographic Data Centre (CCDC; Cambridge, UK) where the technique is applied to the problem of docking ligands to protein binding sites.

Accurate ligand docking, typically of actual or potential drug molecules, to proteins is one of the most important unsolved problems in computer-aided drug design. If a ligand is flexible, picking its single active bound conformation out of the huge range of possible conformations is an immensely complex and time-consuming problem. The traditional docking approach predicts the structure and binding free energy of a ligand-receptor complex using the structures of the free ligand and receptor. With the development of docking approaches that scan hundreds of three-dimensional structures in public databases, the need for more efficient algorithms has become more pressing.

This type of problem-searching a manydimensional conformational space for a small subset of possible solutions-is an ideal application for a genetic algorithm. These use simulated Darwinian evolution to obtain rapid and accurate solutions to complex searches.

Clare Sansom is a freelance writer working in Reading, UK.
A typical run of GOLD takes 30 minutes to a few hours (depending on the flexibility of the ligand) much less than the computationally demanding conformational searching programs. GOLD represents each protein-ligand complex as a set of "chromosomes" ("artificial creatures" composed simply of strings of characters). These are subjected to random "mutations" and "crossovers"-analogous to the respective processes in sexual reproduction - to produce daughter chromosomes which are tested against a fairly standard energy function. Eventually, an initial random population of chromosomes will converge onto a set of the "fittest" structures-which should, in theory, correspond to the optimum conformation of the protein-ligand complex.

GOLD has passed the classic test of docking algorithmspredicting the conform- ational "flip" between methotrexate and folate docked to dihydrofolate reductase-with flying colors. In a much more rigorous test, Robin Taylor at the CCDC used it to predict the binding conformations of an unbiased set of 100 complexes taken from the Protein Data Bank (PDB; Brookhaven, NY). The results were compared against the actual conformations and assessed subjectively as "good"; "close"; "significant errors"; or "just plain wrong". Counting the first two classes as "successes", the program now has a success rate of $75 \%$, as good as commercially available knowledge-based or conformational search methods. Although it is unlikely that this will be repeated in a real drug design situation, as drug molecules show more structural diversity than ligands found in the PDB, GOLDis still likely to become a very useful tool. 